\title{
BÚSQUEDA DE AYUDA EN FOROS ELECTRÓNICOS. FRECUENCIA $Y$ CONTENIDO DE LAS PREGUNTAS FORMULADAS POR ESTUDIANTES DE GRADO Y POSGRADO
}

\author{
(HELP SEEKING IN ELECTRONIC FORUMS. STUDENT'S QUESTIONS FREQUENCY AND \\ CONTENT)
}

Analía Chiecher

Danilo Donolo

María Cristina Rinaudo

Universidad Nacional de Río Cuarto (Argentina)

\section{RESUMEN}

Las conductas de búsqueda de ayuda por parte de los estudiantes han sido objeto de interés de investigadores diversos. Más recientemente, se advierte preocupación por atender al modo en que se presentan estas conductas en ambientes virtuales. El propósito de este artículo es analizar la frecuencia y el contenido de las intervenciones de búsqueda de ayuda realizadas por dos grupos de estudiantes que aprendían en ambientes virtuales. Uno de los grupos cursaba estudios de grado bajo una modalidad mixta $(\mathrm{N}=48)$; el otro grupo, cursaba estudios de posgrado a distancia $(\mathrm{N}=20)$. Se tomaron como objeto de análisis las intervenciones realizadas por estos estudiantes en foros habilitados especialmente para el pedido de asistencia. Los resultados se presentan en términos de los propósitos de las solicitudes de ayuda registradas en ambos grupos, principalmente vinculadas con cuestiones técnicas y esclarecimiento de consignas y, llamativamente, poco dirigidas al esclarecimiento de conceptos.

Palabras clave: búsqueda de ayuda, contextos virtuales, estrategias de regulación de recursos.

\begin{abstract}
Students' help-seeking behaviours have been the object of interest for diverse researchers. More recently, attention has been focused on help-seeking in virtual contexts. The purpose of this article is to
\end{abstract}


report the analysis of the content and frequency of help-seeking behaviours by two groups of students in virtual contexts. One group was engaged in undergraduate studies via a mixed modality $(\mathrm{N}=48)$; the other group was engaged in master's level studies in a distance learning modality $(\mathrm{N}=20)$. The focus of this study was the activities of students in forums designed to promote help-seeking behaviors. The findings are presented in terms of the purposes of the requests for help made by both groups, principally those related to technical questions and to clarification of instructions.

Keywords: help seeking, virtual contexts, management resources strategies.

\section{LA CONDUCTA DE BÚSQUEDA DE AYUDA}

\section{Avances conceptuales en su estudio}

Las conductas de búsqueda de ayuda por parte de los estudiantes han sido objeto de interés y de estudio por parte de investigadores diversos, sobre todo desde 1980 en adelante (Butler, 1998; Butler y Neuman, 1995; Karabenick, 1998 y 2002; NelsonLe Gall, 1985; Newman, 1998; Newman y Schrwager, 1995; Rinaudo et al., 1999; Ross y Cousins, 1995; Ryan et al., 2001).

Cuando los alumnos son incapaces de resolver un problema, entender un texto o comprender las explicaciones del docente, sus opciones incluyen no sólo los extremos de persistir hasta el cansancio por sí mismos o abandonar directamente la tarea, sino que también entra en el abanico de posibilidades la de obtener asistencia de fuentes diversas, tales como los compañeros, amigos o profesores (Karabenick, 1998). En efecto, la búsqueda de ayuda es considerada como una estrategia de regulación de recursos del ambiente (Pintrich et al., 1991), que implica la formulación de una inquietud, duda o pedido, dirigida al docente o a un compañero, a fin de obtener una respuesta que contribuya a dar solución a un problema o dificultad percibida como tal.

Si bien la importancia de los procesos sociales para el aprendizaje fue destacada desde hace tiempo, fue más recientemente -quizás desde la década de los 80- que la búsqueda de asistencia o ayuda comenzó a ser considerada como significativa y relevante para el aprendizaje. Previamente, aquellos que buscaban ayuda eran caratulados como dependientes y, por tanto, como la antítesis de lo que se esperaba como criterio de excelencia (Karabenick, 1998). Como anticipábamos, esta visión ha cambiado radicalmente al reconocer el valor estratégico de la búsqueda de ayuda 
para el aprendizaje (Arroyo et al., 2004; Butler, 1998; Karabenick, 1998 y 2002; Kitsantas y Chow, 2007; Melrose et al, 2005; Nelson-Le Gall, 1985; Newman, 1998; Newman y Schrwager, 1995; Rinaudo et al, 1999; Ross y Cousins, 1995; Ryan et al, 2001; Pintrich et al, 1991; Tapin et al, 2001).

$\mathrm{Al}$ referir al valor de la búsqueda de ayuda para la construcción de conocimientos no podemos soslayar importantes aportes relacionados con: a) la identificación y descripción de distintas etapas incluidas en el proceso de buscar ayuda; b) los distintos tipos de búsqueda de ayuda; c) los diversos factores -tanto personales como contextuales- que inciden en la activación de los mecanismos y modalidades de pedir asistencia. Referiremos a estas cuestiones en los próximos apartados. Atenderemos en lo sucesivo a las tres cuestiones mencionadas.

\section{Etapas en el proceso de buscar ayuda}

Para iniciar el proceso de búsqueda de ayuda el alumno debe tomar conciencia de que está enfrentando un obstáculo que le impide alcanzar su meta, debe poder ver a otras personas como recursos valiosos en la búsqueda de una solución, debe ser hábil en atraer y convencer a esas personas para que le provean de la asistencia necesaria y luego debe saber usar la información que recibe. Por ello, aunque beneficiosos para el aprendizaje, los procesos de solicitar ayuda transcurren en contextos educativos, pero no exentos de obstáculos e interrupciones.

La conciencia de la necesidad de ayuda -primera de las etapas del proceso- es esencial para su inicio. Si los sujetos tienen conciencia de la complejidad de la tarea o del contenido a aprender, pueden monitorear su proceso de aprendizaje y detectar los problemas, están en buenas condiciones de usar la búsqueda de ayuda como estrategia para lidiar con ellos y superarlos (Nelson Le-Gall, 1985; Van der Meij, 1990). Según Nelson Le-Gall (1985), esta capacidad de ser conciente y de monitorear el propio proceso de aprendizaje se incrementa con el desarrollo; de este modo, los adultos o aprendices maduros tendrían mayor conciencia de las dificultades que experimentan mientras aprenden y, en tal sentido, estarían en condiciones de formular pedidos de ayuda más específicos que los niños.

Una vez percibido el problema, debe ser resuelto de manera apropiada; esto es, el sujeto debe poder tomar la decisión de buscar ayuda. Así también debe ser capaz de formular un pedido de ayuda que verdaderamente contribuya a su progreso en el aprendizaje de un contenido o en la resolución de una tarea. Se ha visto que algunos estudiantes prefieren recibir una ayuda instrumental (por ejemplo, sugerencias, 
explicaciones, ejemplos elaborados) y otros se inclinan por recibir ayuda que directamente le permita completar la tarea (por ejemplo, respuestas directas). También se ha demostrado ampliamente que el primer tipo de búsqueda de ayuda, la instrumental, es el que más beneficios reporta al aprendizaje (Karabenick, 2002; Nelson Le-Gall, 1985; Newman, 1998; Newman y Schrwager, 1995; Ryan et al, 2001; Taplin et al, 2001).

Habiendo tomado la decisión de buscar ayuda, el alumno debe ser capaz de identificar a la persona adecuada para pedírsela. En efecto, un aspecto importante es el reconocimiento de que no todas las personas están en las mismas condiciones para ofrecer la ayuda necesaria (Nelson Le-Gall, 1985). Una vez identificada la persona, el estudiante tiene que usar estrategias que convenzan al destinatario de ofrecerle la ayuda pedida; para ello pueden utilizarse estrategias verbales -como pedirle directamente la ayuda, solicitar información sobre el problema, preguntar por sus capacidades sobre el asunto- y estrategias no verbales -tales como aproximarse a la persona, tomar contacto visual o usar expresiones físicas que denoten confusión(Nelson Le-Gall, 1985).

Aún recibida la ayuda, el proceso no ha terminado puesto que tiene que realizarse una evaluación de la respuesta obtenida en términos de su ajuste y adecuación al problema experimentado, debe hacerse un uso adecuado de la misma o bien insistir en el pedido de ayuda si la asistencia recibida no responde enteramente a la dificultad percibida en principio (Nelson Le-Gall, 1985; Ross y Cousins, 1995).

Como puede notarse, el proceso de solicitar, recibir y saber usar ayuda pedagógica reviste cierta complejidad y no se da siempre exento de turbulencias.

\section{Tipos de búsqueda de ayuda}

Uno de los pioneros en proponer una distinción entre distintos tipos de búsqueda de ayuda fue Nelson-Le Gall (1985); distinción que ha sido ampliamente aceptada en la literatura que reporta estudios e investigaciones sobre el tema y que aluden a la diferenciación entre dos tipos de búsqueda de ayuda: a) la búsqueda de ayuda ejecutiva u orientada a la dependencia $\mathrm{y} b$ ) la búsqueda de ayuda instrumental $\mathrm{u}$ orientada al dominio de la tarea.

La búsqueda de ayuda ejecutiva refiere a instancias en las que la intención del estudiante es conseguir que alguien le resuelva el problema. Se trata de sujetos que parecen estar más interesados en el producto y en obtener un resultado exitoso que 
en el proceso de lograr determinado resultado; por lo tanto, ubica la responsabilidad en quien proporciona asistencia, demandando fundamentalmente una ayuda directa y soluciones hechas que le permitan reducir el tiempo y esfuerzo que le demandaría resolver por sí mismo el problema (Kitsantas y Chow, 2007; Melrose et al., 2005; Nelson-Le Gall, 1985; Ryan et al., 2001; Taplin et al., 2001).

En cambio, la búsqueda de ayuda instrumental está orientada hacia el dominio de la tarea y refiere a instancias en las que el pedido de ayuda se focaliza en adquirir procesos exitosos de resolución de problemas. En este caso, la responsabilidad se ubica en quien busca ayuda y la asistencia solicitada suele limitarse a la cantidad y tipo necesarios para poder resolver el problema por uno mismo. Por lo tanto, las respuestas que ofrecen ayudas indirectas, sugerencias y explicaciones son de interés para este tipo de búsqueda de ayuda (Kitsantas y Chow, 2007; Melrose et al., 2005; Nelson-Le Gall, 1985; Ryan et al., 2001; Taplin et al., 2001).

Como parece lógico, los pedidos de ayuda instrumental están asociados más fuertemente con los beneficios cognitivos que se ha informado puede reportar el uso adecuado de esta estrategia de buscar ayuda.

\section{Factores que inciden en la búsqueda de ayuda}

El acto de pedir ayuda es un fenómeno complejo y altamente dependiente de factores tanto personales como contextuales (Kitsantas y Chow, 2007; Nelson LeGall, 1985; Newman, 1998; Ryan et al., 2001).

Entre los factores personales que se ha estudiado afectan la búsqueda de ayuda, se pueden mencionar las percepciones y creencias de los estudiantes, su edad, el logro previo, la autoestima, las metas que persiguen, etc.

Las percepciones de competencia cognitiva -o autoeficacia- y la autoestima que cada estudiante tiene sobre sí mismo afectan considerablemente los procesos de búsqueda de ayuda. En efecto, se ha visto que la necesidad de asistencia es considerada como una amenaza por estudiantes de bajo rendimiento o con una autoestima pobre, puesto que asocian esta necesidad con una falta de competencia o incapacidad que ponen en evidencia frente a otros. En cambio, los alumnos con rendimientos más altos y con percepciones positivas acerca de sus propias competencias cognitivas se muestran menos preocupados por el hecho de que otros atribuyan su necesidad de ayuda a una falta de habilidad y entonces están más dispuestos a pedirla cuando la creen necesaria (Kitsantas y Chow, 2007; Nelson Le- Gall, 1985; Ryan et al., 2001). 
Una relación similar se ha observado entre las percepciones de competencia social y los procesos de búsqueda de ayuda. En efecto, la búsqueda de ayuda no sería solamente una estrategia de autorregulación relacionada con la esfera de lo cognitivo sino también con la interacción social. De este modo, los estudiantes que se sienten más cómodos y se saben socialmente habilidosos en relación con los otros, muestran también una mayor confianza en su destreza para pedir ayuda sin esperar una reacción negativa de parte del otro (Ryan et al., 2001)

Otro de los factores ampliamente vinculado con los procesos de buscar ayuda ha sido el de las metas hacia las que se orientan los estudiantes. En este aspecto, existe amplio consenso en considerar que resulta más probable que los sujetos se dispongan a pedir ayuda cuando persiguen metas de aprendizaje y no de desempeño (Butler y Neuman, 1995; Karabenick, 2002; Kitsantas y Chow, 2007; Nelson Le-Gall, 1985; Newman, 1998; Newman y Schrwager, 1995; Ryan et al., 1995). Esto es, los estudiantes más activos, comprometidos y autorregulados están más motivados y por tanto más dispuestos a buscar ayuda si la creen necesaria.

Otro de los factores personales que se ha sugerido incide en la búsqueda de ayuda es el de la edad o nivel de desarrollo de los sujetos. En esta línea, algunas investigaciones sugieren que los chicos más pequeños tienen mayores dificultades para evaluar la necesidad de ayuda; ello puede deberse a que sus habilidades metacognitivas están todavía menos desarrolladas. Pareciera que mientras más conocimiento y experiencia tienen los sujetos, más suelen reconocer la necesidad de ayuda (Nelson-Le Gall, 1985; Newman y Schrwager, 1995; Puustinen, 1998; Ryan et al., 2001).

En cuanto a los factores del contexto que se ha informado afectan la decisión de buscar ayuda, se encuentran las características de la propuesta instruccional, el clima y las reglas de la clase, la apertura y flexibilidad del docente, la estructura de metas de la clase, etc.

Las reglas y normas que los docentes establecen en clase inciden sobre las oportunidades para pedir ayuda. Por ejemplo, algunos profesores permiten el diálogo entre pares durante la resolución de una tarea -lo que facilita el pedido de ayudamientras que otros no (Ryan et al., 2001). También las cualidades personales del docente pueden influenciar sobre la búsqueda de ayuda (Kitsantas y Chow, 2007).

Otro factor contextual importante lo constituyen las metas que orientan la tarea. La estructura de la clase puede estar orientada hacia el aprendizaje o hacia 
el desempeño. Una clase orientada hacia metas de dominio o de aprendizaje está organizada de tal modo de dar a entender a los estudiantes que las razones para comprometerse en una tarea tienen que ver principalmente con el aprendizaje y su valor intrínseco. En cambio, una clase orientada hacia el desempeño pone en primer plano la demostración de habilidad y la comparación respecto de los otros. Distintos estudios han informado que los estudiantes se muestran más dispuestos a participar cuando perciben que la clase se orienta hacia el aprendizaje (Kitsantas y Chow, 2007; Nelson Le-Gall, 1985; Newman, 1998; Newman y Schrwager, 1995; Ryan et al., 2001).

Por fin, el clima social e interpersonal de la clase, la familiaridad y las relaciones positivas incrementan la disposición del estudiante para pedir ayuda a otra persona (Ryan et al., 2001; Kitsantas y Chow, 2007).

\section{NUEVAS TENDENCIAS}

\section{El estudio de la búsqueda de ayuda en ambientes virtuales}

Con la aparición y proliferación de los contextos educativos mediados por tecnologías parecen reeditarse problemas e interrogantes referidos a la enseñanza y al aprendizaje que ya han sido estudiados en el marco de contextos presenciales, pero que requieren ahora de una reconsideración, a la luz de las características tan particulares de los nuevos entornos virtuales.

Uno de los temas que claramente ha despertado interés es el de las interacciones profesor-alumno y alumno-alumno en contextos virtuales donde, a diferencia de los presenciales, la relación entre los participantes no es cara a cara. Y dentro de este tema más general, un tipo particular de intercambio, el de buscar y recibir ayuda, ha sido objeto de atención de investigadores diversos en los últimos años (Blocher y Sujo, 2002; Blocher et al., 2002; Chiecher et al., 2006 y 2007; Keefer y Karabenick, 1998; Kitsantas y Chow, 2007; Melrose et al., 2005; Taplin et al., 2001; Suárez y Anaya, 2004; Tucker y Blocher, 2002).

Blocher y Sujo (2002), Blocher et al. (2002) y Tucker y Blocher (2002) se preguntan si los estudiantes a distancia tienen perfiles específicos que pueden hacer que el aprendizaje colaborativo sea menos atractivo para ellos. En efecto, resultados de sus investigaciones informaron que el aprendizaje con pares es la estrategia menos usada por estos alumnos. Además, cuando necesitan ayuda, prefieren solicitarla 
al docente y, en segundo lugar, a sus pares. Ellos interpretan que, tal vez, años de escolaridad han marcado esta idea de que hacer las cosas solo es mejor que en grupo; o bien, que al verse trabajando solos detrás de una computadora entienden que no deben entonces contar con sus pares.

En la misma línea de intereses, Kisantas y Chow (2007) notaron la necesidad de analizar la búsqueda de ayuda en contextos instruccionales tradicionales, mixtos (tradicionales que combinan el uso de tecnologías) y a distancia. Sus hipótesis de partida sostenían que los estudiantes de cursos que incluían alguna instancia mediada por tecnologías: (a) mostrarían mayor disposición para pedir ayuda, particularmente dirigida a lo profesores; (b) se sentirían menos amenazados que los estudiantes en ambientes tradicionales y (c) preferirían medios electrónicos para canalizar la búsqueda de ayuda. Cabe señalar, que estas presunciones iniciales fueron avaladas por los datos del estudio.

Por su parte, otro grupo de investigadores (Melrose et al., 2005) se interesaron en estudiar las experiencias de búsqueda de ayuda en un grupo de estudiantes de posgrado de disciplinas vinculadas a la salud que aprendían en un ambiente virtual. En este caso, la investigación estuvo guiada por tres preguntas: (a) ¿creen necesitar ayuda los estudiantes online de disciplinas referidas al cuidado de la salud?; (b) ¿qué estrategias implementan para buscar ayuda?; (c) ¿qué tipo de problemas ocurren durante el proceso de buscar ayuda? Los resultados del estudio son presentados en términos de cuatro temas que emergieron como importantes:

- Sin excepción, todos los estudiantes señalaron leer y releer los materiales del curso antes de buscar ayuda.

- Consideraron que los propios compañeros son una fuente primaria de ayuda. En efecto, cuando no logran resolver por sí mismos la dificultad mediante la reflexión y relectura, consideran que sus compañeros son una fuente de ayuda.

- Consideraron que implicar a la familia, amigos y compañeros de trabajo provee un soporte educacional.

- Destacaron la importancia del primer mensaje del instructor, su implicación en las discusiones y los comentarios de anécdotas o cuestiones personales. En efecto, valoraron como significativo el diálogo con el instructor y afirmaron que el tono de los mensajes y la rapidez en responder constituyen indicadores de la posibilidad de conseguir o no alguna ayuda. 
Taplin et al. (2001) se interesaron también por analizar la búsqueda de ayuda en ambientes de educación a distancia. Con ese propósito, compararon las conductas de búsqueda de ayuda de dos grupos, uno de alto rendimiento y uno de bajo rendimiento. $\mathrm{Si}$ bien no se encontraron en general diferencias estadísticamente significativas entre los grupos, informan que la mayoría de los estudiantes consideraron a la búsqueda de ayuda como una buena manera de aprender. Sin embargo, al igual que los estudiantes que participaron del estudio de Melrose et al. (2005), creen también que en primera instancia deben intentar solucionar por ellos mismos la dificultad.

Suárez y Anaya (2004) trabajaron con dos grupos de estudiantes universitarios; uno de los grupos aprendía bajo modalidad presencial y el otro con modalidad virtual. Hallaron diferencias significativas que favorecen a los estudiantes en la modalidad de educación presencial respecto del uso de estrategias de aprendizaje colaborativo, es decir, con otros compañeros. No se obtuvieron en este estudio diferencias significativas respecto a las estrategias de búsqueda de ayuda desplegadas en ambos contextos.

En nuestros estudios sobre el tema (Chiecher, 2007; Chiecher et al., 2006 y 2007) hemos trabajado comparativamente con diferentes grupos de estudiantes bajo modalidad presencial y virtual. Se evaluó el uso de distintas estrategias de regulación de recursos y entre ellas la búsqueda de ayuda. Los resultados informaron diferencias significativas entre los grupos, favorables siempre al grupo presencial. Los estudiantes parecen estar más dispuestos a pedir ayuda en las clases y no en ambientes electrónicos. Pensamos que una posible interpretación de estos resultados tiene que ver con el hecho de que el estudiante en un ambiente virtual se siente más solo, intenta aprender y resolver problemas de modo más independiente $\mathrm{y}$, en consecuencia, tiene menor inclinación a buscar la ayuda que otros pudieran brindarle.

En síntesis, inicialmente podría esperarse que en el ambiente online algunas barreras que actúan como obstaculizadoras del pedido de ayuda en contextos tradicionales serían atenuadas. Por ejemplo, una persona introvertida probablemente se disponga mejor a pedir ayuda -y se sienta menos amenazada- en un ambiente virtual, donde están ausentes las presiones sociales que existen en la interacción cara a cara (Kitsantas y Chow, 2007). Sin embargo, los resultados de distintos estudios muestran que en muchos casos las diferencias esperadas no se hacen evidentes, o bien, se aprecian diferencias pero en el sentido opuesto al esperado; es decir, los estudiantes informan estar más dispuestos a pedir ayuda en el ambiente presencial y no en el virtual. Por nuestra parte, pensamos que esta menor predisposición de los alumnos para solicitar ayuda en ambientes virtuales puede relacionarse con la 
mayor autonomía, independencia y -por qué no soledad- que experimentan en este tipo de ambientes de aprendizaje. En estas condiciones, al toparse con un problema puede que primero intenten resolverlo solos y recién después, si esa instancia resulta infructuosa, decidan recurrir al pedido de ayuda.

De todos modos, coincidimos con Keefer y Karabenick (1998) cuando plantean que si bien el impacto de las TIC en el aprendizaje es objeto de continuo debate, su influencia sobre los procesos de búsqueda de ayuda no ha sido aun suficientemente analizada.

\section{PROPÓSITOS}

Habiendo destacado la importancia de estudiar las conductas de búsqueda de ayuda en ambientes virtuales, en este artículo nos proponemos analizar la frecuencia y el contenido de las intervenciones de búsqueda de ayuda realizadas por dos grupos de estudiantes que aprendían en ambientes virtuales, pero en condiciones algo diferentes. En el siguiente apartado describimos con mayor detalle los grupos estudiados y los instrumentos usados para la recolección de los datos.

\section{METODOLOGÍA}

Se tomó como material de análisis las intervenciones que realizaron dos grupos de alumnos en foros habilitados especialmente para la formulación de dudas y consultas.

Los dos grupos -en adelante Grupo 1 y Grupo 2- tienen características diferentes, lo cual nos hizo sospechar que las conductas de búsqueda de ayuda podrían diferir en uno y en otro.

El Grupo 1 está conformado por 48 estudiantes que cursaron una asignatura del plan de estudios de la Licenciatura en Psicopedagogía en la Universidad Nacional de Río Cuarto (Argentina). Estos alumnos, el 100\% mujeres, informaron una edad promedio de 20 años. En cuanto al estado civil, el $98 \%$ son solteros. La tarea de estudiar es una actividad primaria para ellos puesto que un $85 \%$ no trabaja, sino que dedica su tiempo exclusivamente a la los estudios universitarios. En este caso, el dictado de la asignatura se apoyó en una modalidad mixta; esto es, los alumnos asistieron a clases presenciales dos veces a la semana y además debieron atender 
a una instancia virtual, soportada en la plataforma SIAT (Sistema Informático de Apoyo a la Teleformación). Dentro de la plataforma pueden crearse foros temáticos; uno de ellos fue habilitado especialmente para canalizar dudas y consultas sobre la asignatura.

El Grupo 2 está integrado por 20 alumnos que cursaron un seminario de posgrado en el marco de una Maestría dictada con modalidad a distancia desde la Universidad Nacional del Comahue (Argentina). En este caso, la edad promedio del grupo es de 40 años; un $75 \%$ son mujeres y el resto varones. $\mathrm{El} \mathrm{100 \%} \mathrm{de} \mathrm{los} \mathrm{sujetos} \mathrm{de} \mathrm{este} \mathrm{grupo}$ trabaja además de dedicar tiempo al estudio y tienen también obligaciones familiares, puesto que un 95\% está en condición de casado. Este grupo cursa sus estudios de posgrado con modalidad a distancia, soportada en la plataforma Moodle. También en esta oportunidad, se habilitó desde el inicio del curso un foro para atender a dudas y consultas de los estudiantes.

Complementariamente se tomaron en cuenta los puntajes obtenidos en la escala relativa a la búsqueda de ayuda del cuestionario MSLQ (Pintrich et al., 1991). El mencionado instrumento incluye una escala conformada por 4 ítems que aluden a la disposición del estudiante para pedir ayuda al docente y a sus pares. Sobre la base de los puntajes obtenidos por los sujetos en esta escala en particular se seleccionaron los casos extremos de ambos grupos (el 1 y el 2) a quienes se administró una entrevista digitalizada a fin de conocer cuestiones tales como el tipo de preguntas que realizaban, los motivos por los que decidían buscar o no ayuda, etc.

\section{RESULTADOS}

Presentaremos los resultados para el Grupo 1 y el 2. Aludiremos en primer término a la frecuencia de los pedidos de ayuda, para referir luego al contenido de las demandas así como a algunas razones que impulsan o inhiben el inicio del proceso.

\section{Grupo 1. Los alumnos de grado}

Frecuencia de los pedidos de ayuda

De los 48 sujetos del Grupo 1 solamente 27 alumnos (56\%) han realizado algún tipo de intervención en el foro habilitado durante el cursado de la materia para atender dudas, preguntas y dar respuestas a inquietudes. Los restantes 21 no intervinieron en ninguna oportunidad. 
El foro analizado tiene un total de 104 intervenciones; de estas, 58 (56\%) fueron efectuadas por los estudiantes y 46 fueron concretadas por los docentes de la asignatura.

Dentro de las 58 intervenciones concretadas por los alumnos, se han hallado 45 pedidos de ayuda; es decir, intervenciones realizadas con el propósito explícito de solicitar información que contribuya a resolver una dificultad conceptual, procedimental o técnica que el estudiante no puede resolver por sí mismo.

\section{Contenido de los pedidos de ayuda}

$\mathrm{Al}$ analizar los contenidos sobre los cuales los estudiantes solicitan aclaraciones, se pueden diferenciar cinco tipos de búsqueda de ayuda: 1) pedido de confirmación de recepción de trabajos; 2) pedido de ayuda sobre cuestiones técnicas; 3) pedido de ayuda para esclarecer consignas de trabajo; 4) pedidos de ayuda sobre la localización de bibliografía del curso; 5) pedidos de ayuda para esclarecer aspectos conceptuales.

Los pedidos de confirmación de recepción de trabajos fueron los más frecuentes. Se registraron en el foro 18 intervenciones con este propósito; es decir, un $40 \%$ del total de pedidos de ayuda. Un ejemplo de este tipo de intervenciones es el siguiente:
Alumno. Profesora: yo quisiera saber si le han llegado correctamente la serie de preguntas que debía responder acerca del manejo de la plataforma y del cursado de la materia... espero su respuesta... desde ya muchas gracias!i

Los pedidos de ayuda sobre cuestiones técnicas fueron 12 y representan un $27 \%$ del total de solicitudes planteadas. Un ejemplo del contenido tipo de estos intercambios es el siguiente:

Alumno. Hola profe!!! Intento subir el cuestionario y me dice que el plazo ha vencido. Quisiera saber cómo tengo que hacer para subirlo a la plataforma!!

Gracias.

Por su parte, los pedidos de ayuda para esclarecer consignas de trabajo fueron menos frecuentes todavía. Se registraron 10 intervenciones de este tipo, que representan un $22 \%$ del total de solicitudes de ayuda. El siguiente es un ejemplo ilustrativo de este tipo de intercambios: 


\begin{abstract}
Alumno. Hola, mis compañeras y yo debemos rehacer la Actividad 1 y no entendemos correctamente el punto 1 donde debemos: mirar la bibliografía listada en el programa y consignar qué materiales responden a esta unidad en particular... de donde sacamos la bibliografía? porque desde Internet no la encontramos... cuando la encontremos ¿debemos colocar el nombre de la bibliografía que corresponde a cada punto de la unidad? o comentar algo sobre eso...
\end{abstract}

Espero su respuesta...gracias!

Los pedidos de ayuda para localizar bibliografía del curso fueron 4; es decir, un 9\% del total de las solicitudes de ayuda concretadas en el foro. Un ejemplo de este tipo de demanda puede verse en el siguiente fragmento:
Alumno. Hola profe!!! Necesitamos saber sonde encontrar los contenidos de los textos de la unidad 2 y 7 ya que buscamos en el cd, también en internet y no encontramos nada!... muchas gracias!

Finalmente, los pedidos de ayuda destinados a esclarecer aspectos conceptuales fueron muy escasos en el marco de este foro; en efecto, solamente se registró una intervención de este tipo ( $2 \%$ del total de solicitudes de ayuda):

\begin{abstract}
Alumno. Hola profe, buenas tardes!! Tengo una duda acerca de la actividad de la unidad número siete... a qué se refiere específicamente con "técnicas proyectivas" y "diagnóstico operativo", porque se nos dificulta encontrar en las carpetas de materiales los archivos que hacen referencia específicamente a estos puntos.
\end{abstract}

Gráficamente, y a modo de síntesis, la siguienteilustración presenta la distribución de frecuencias de las solicitudes de ayuda conforme a su contenido.

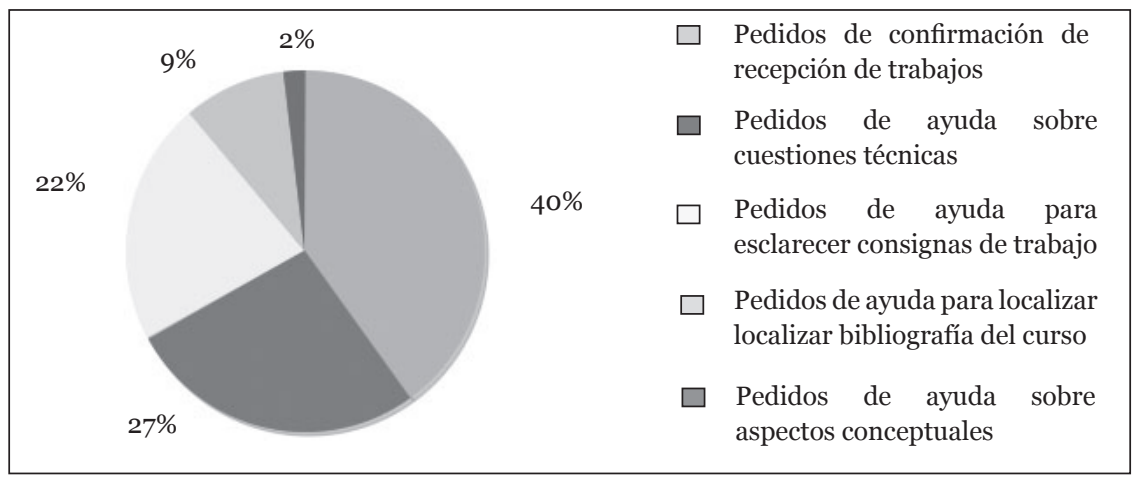

Gráfico 1. Frecuencia de distintos tipos de búsqueda de ayuda en estudiantes de grado 
En el apartado siguiente procuraremos atender a algunos de los motivos que impulsan a los estudiantes a buscar ayuda y a aquellos otros que inhiben la concreción de esta conducta.

Razones que impulsan la búsqueda de ayuda y motivos que la inhiben

Como se anticipó en la sección metodológica, además de observar y analizar los mensajes colocados en los foros, se administró un cuestionario de preguntas abiertas a los sujetos que obtuvieron puntajes extremos en la escala relativa a la búsqueda de ayuda del MSLQ.

Para este grupo de estudiantes de grado, el puntaje inferior en la escala (que puede variar entre 4 y 28) fue de 11 puntos y el puntaje más alto de 27.

Tres sujetos obtuvieron 11 puntos, indicativos de una baja disposición para pedir ayuda a otros, en tanto que un sujeto informó una alta disposición para solicitar ayuda, sumando 27 puntos en la escala.

El estudiante que obtuvo el máximo puntaje manifestó no haberse quedado durante el cursado de la asignatura con preguntas sin formular; es decir, según su testimonio, cuando experimentaba una duda estaba dispuesto a ponerla en consideración de otro para obtener así la ayuda necesaria.

Por su parte, los tres sujetos que obtuvieron en la escala el puntaje más bajo del grupo manifestaron no realizar preguntas en el contexto de la plataforma por razones diversas; entre ellas, preferían consultar en las clases presenciales, se sacaban las dudas con sus compañeros, experimentaban sentimientos de introversión, etc. Se transcriben a continuación los testimonios de estos sujetos:

Alumno 1. "Las veces que no formulé preguntas al profesor es porque me saqué las dudas con mis compañeras".

Alumno 2. "Para sacarme las dudas siempre prefería hacerlo en clases presenciales o les preguntaba a mis compañeras en otras clases o en momentos libres"...

Alumno 3. "A veces me quedé con preguntas sin formular... no se bien el motivo, quizás por vergüenza o porque no me parecían preguntas interesantes". 
Cabe señalar que al analizar las intervenciones realizadas en el foro, no se reflejó una mayor cantidad de contribuciones de parte del sujeto que informó una mayor disposición para pedir ayuda y, a la inversa, intervenciones escasas de quienes obtuvieron los puntajes más bajos en la escala. Sin embargo, sí parece apreciarse alguna diferencia respecto de las razones y motivos que esgrimen para justificar su disposición hacia la búsqueda de ayuda. En efecto, el estudiante con puntaje más alto, aunque no ha formulado abundantes intervenciones, dice no haberse quedado con cuestiones pendientes; esto es, no ha participado porque no ha experimentado la necesidad de pedir ayuda. En cambio, los estudiantes que obtuvieron los puntajes más bajos han sentido la necesidad de recibir ayuda pero, a pesar de ser concientes de ello, tomaron la decisión de no exponer sus demandas, al menos en el ambiente virtual.

\section{Grupo 2. Los alumnos de posgrado}

Frecuencia de los pedidos de ayuda

De los 20 sujetos del Grupo 2, un 80\% (16 estudiantes) ha participado al menos una vez en el foro habilitado durante el cursado de la materia para atender dudas, preguntas y dar respuestas a inquietudes. Los restantes 4 alumnos no intervinieron en ninguna oportunidad.

El foro analizado tiene, coincidentemente con el anterior, un total de 104 intervenciones; de estas, 64 (62\%) fueron efectuadas por los estudiantes y 40 (38\%) fueron concretadas por los docentes de la asignatura.

Dentro de las 64 intervenciones concretadas por los alumnos, se han hallado 34 pedidos de ayuda.

\section{Contenido de los pedidos de ayuda}

Para este grupo, las categorías relativas a los tipos de solicitudes de ayuda así como la frecuencia con que se presentó cada uno mostraron algunas variaciones en comparación con el grupo anterior. En efecto, se encontró que los pedidos de ayuda de estos alumnos referían a: 1) pedido de ayuda sobre cuestiones técnicas; 2) pedido de ayuda para esclarecer consignas de trabajo; 3) consultas sobre reprogramación de tareas en casos en que no se pudieron entregar en término; 4) pedido de confirmación de recepción de trabajos; 5) pedidos de ayuda sobre la localización de bibliografía del curso; 6) pedido de ayuda sobre conceptos. 
Los pedidos de ayuda sobre cuestiones técnicas fueron uno de los más frecuentemente concretados; se registraron 9 mensajes con este contenido (28\% de las solicitudes de ayuda). El siguiente es un ejemplo ilustrativo de este tipo de solicitud: Alumno. Tengo una consulta. Cómo selecciono un nuevo tema dentro del
foro del grupo 1. No encuentro el hipervínculo para hacerlo.

Los pedidos de ayuda destinados a esclarecer consignas se presentaron con igual frecuencia que la categoría anterior; esto es, el foro analizado contiene 9 mensajes con este propósito (28\% de las solicitudes de ayuda). El siguiente es un ejemplo:

\begin{abstract}
Alumno. La consigna de la actividad $\mathrm{G}$ nos pide formular 3 preguntas centrales sobre metodología de la investigación al capítulo considerado... Mi duda es: ¿debemos concentrarnos en los aspectos metodológicos mostrados por el capítulo elegido? ¿No hay que atender a la temática sino más bien a cómo se presenta la temática? Gracias.
\end{abstract}

Una categoría nueva estuvo representada por las consultas sobre reprogramación de tareas en casos en que no se pudieron entregar en término. En efecto, por las características del curso a distancia y de los participantes implicados, en algunas ocasiones los estudiantes consultaban pidiendo nuevos plazos para ponerse al día con las tareas del curso. Estas intervenciones fueron 6 en el foro (19\% de las solicitudes de ayuda). Un ejemplo de este tipo de intercambio es el siguiente:

\begin{abstract}
Alumno. Estoy muy interesada en hacer esta materia, pero tengo una dificultad, estoy embarazada, mañana tengo mi cesárea, así que no creo que pueda cumplir la tarea $\mathrm{C}$ en el tiempo que uds. han colocado, habrá alguna posibilidad de que la pueda enviar unos días más tarde?
\end{abstract}

$\mathrm{Al}$ igual que en el grupo anterior, también se hicieron presentes en este caso pedidos de confirmación de recepción de trabajos. Se registraron 5 solicitudes de este tipo (16\% de las solicitudes ayuda). A continuación un ejemplo:

\begin{abstract}
Alumno. Estimados profesores: acabo de subir la actividad B, cuando entregué la actividad A la llamé Tarea 1, espero que ambas hallan llegado sin dificultad ya que cuando entregué la anterior no envié correo avisando la entrega ¿Podrían confirmarme la llegada de ambas? Gracias.
\end{abstract}


Se registraron también 3 pedidos de ayuda sobre la localización de bibliografía del curso ( $9 \%$ de las solicitudes de ayuda). El siguiente es un ejemplo:

\begin{abstract}
Alumno. Estuve mirando la bibliografía para realizar la actividad C...Es un documento sobre investigación en educación. En el CD no lo encontré y colgado tampoco, quería saber dónde buscarlo. Gracias.
\end{abstract}

Finalmente, 2 pedidos (6\%) buscaban esclarecer conceptos trabajados en el curso. A continuación un ejemplo:

\begin{abstract}
Alumno. Te hago una consulta sobre uno de los conceptos que estoy leyendo y no logro entender (hay miles que no logro entender pero este es uno muy básico). Cuando hablamos de escalas: entiendo la escala nominal, ordinal, pero luego en uno de los documentos habla de escala intervalo y ratio (las define pero no entiendo bien la diferencia). En otro documento, introduce la escala intervalo y razón. Respecto a "intervalo", tenía entendido que cuando una variable es continua tomamos intervalos para medir las frecuencias, no sé si es así. En cuanto a ratio o razón no sé. Bueno, espero tu respuesta. Gracias!! Saludos.
\end{abstract}

En síntesis, la siguiente ilustración presenta la distribución de frecuencias de las solicitudes de ayuda realizadas por estudiantes de posgrado.

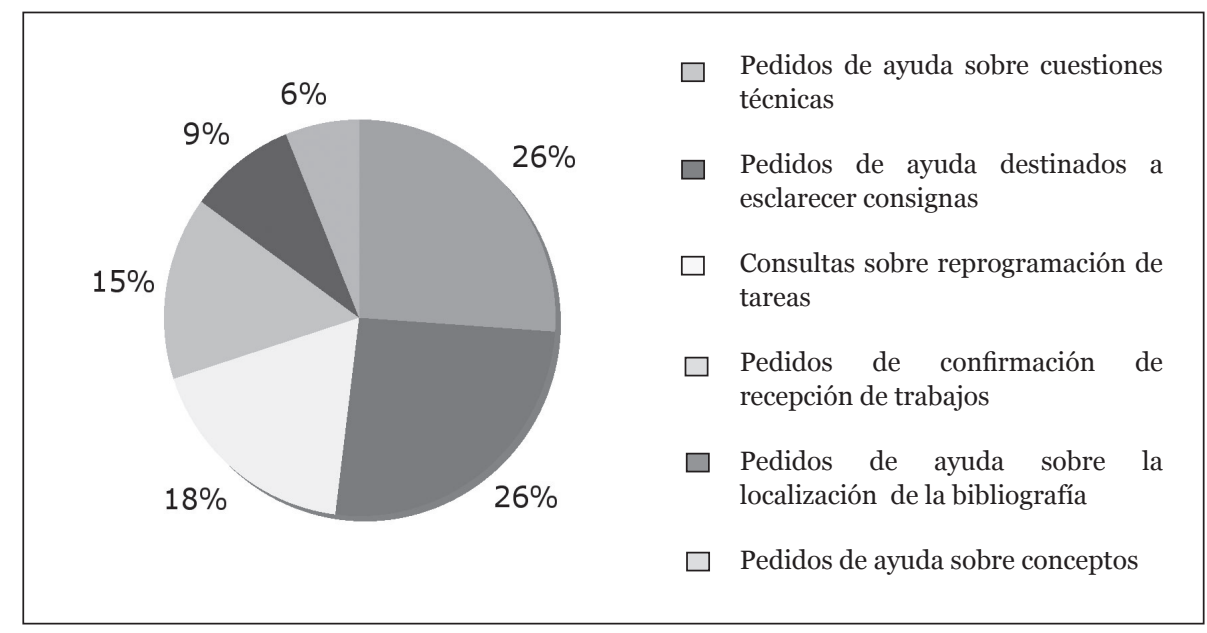

Gráfico 2. Frecuencia de distintos tipos de solicitudes de ayuda para estudiantes de posgrado 
Razones que impulsan la búsqueda de ayuda y motivos que la inhiben

Para este grupo tenemos dos puntajes extremos en la escala relativa a búsqueda de ayuda. El puntaje más alto fue de 24 y el más bajo de 7 .

El estudiante que informó la mayor predisposición para pedir ayuda, no refleja una participación abundante en el foro analizado. Su testimonio indica lo siguiente:

\begin{abstract}
Alumno. "No hice preguntas porque eran las mismas que hacían mis compañeros en los foros"
\end{abstract}

Pareciera que, aunque está dispuesto a pedir ayuda cuando la considera necesaria, en muchas ocasiones las preguntas que realizan sus compañeros -y por supuesto, las respuestas a esas preguntas- le son de utilidad para resolver sus propias dudas. En cambio, el alumno que informó menor disposición para pedir ayuda, argumenta que en realidad no la ha necesitado y por ese motivo no la ha pedido.

\begin{abstract}
Alumno. "Si bien no fue una característica mía preguntar, no tuve mayores inconvenientes en comprender lo que se solicitaba. Si tuve alguna duda pregunté y me fue contestada... quizás a lo que no me animé fue a opinar un poco más sobre cuestiones que surgían en el cursado, pero no tengo muy claro el por qué ya que en contextos presenciales sí participo... la verdad es que a uno nadie lo conoce y no creo que ese fuera el motivo, pienso que puede ser la distancia y el no conocer justamente a los otros compañeros... por ahí me daba ganas de contestar algunas preguntas que hacían, pero finalmente decidía no hacerlo."
\end{abstract}

También pueden apreciarse en su testimonio algunas referencias a rasgos de personalidad que inhibirían su participación en el foro. En ocasiones se sintió capaz de responder a pedidos de ayuda de sus compañeros, pero decidió no hacerlo.

\title{
DISCUSIÓN Y CONCLUSIONES
}

Se tomaron como objeto de análisis las solicitudes de ayuda de dos grupos de estudiantes que aprendían en un ambiente virtual. Los grupos diferían en aspectos importantes, lo que según entendíamos podía incidir en las conductas de pedido de asistencia que observáramos. 
El Grupo 1 -conformado por estudiantes de grado, en general jóvenes y con dedicación exclusiva al estudio- cursaba una asignatura con modalidad tradicional, instancia a la que se sumó la propuesta de atender a tareas en el marco de una plataforma virtual. Por su parte, el Grupo 2 -integrado por estudiantes adultos, en nivel de posgrado, con obligaciones laborales y familiares y, por lo tanto, con dedicación parcial al estudio- cursaba un seminario en el marco de una maestría dictada con modalidad virtual.

Como vimos, algunas variaciones hubo entre los grupos, aunque también se advirtieron algunas similitudes en cuanto a los pedidos de ayuda observados.

En el Grupo 1 abundaron los pedidos de confirmación de la recepción de trabajos y las solicitudes de ayuda acerca de cuestiones técnicas. Pareciera que la falta de experiencia de estos alumnos en ambientes virtuales -sumado al hecho de que no esperaban atender a una instancia mediada por tecnologías- ayudó a configurar en ellos una preocupación centrada en el aspecto tecnológico que opacó, quizás, el aprendizaje que podían obtener de la experiencia. Por su parte, en el Grupo 2 aparecieron también las preocupaciones ligadas al manejo de la tecnología pero en igual medida que los pedidos de ayuda sobre consignas. Analizando las demandas de este grupo, pudo apreciarse una preocupación más clara que en el Grupo 1 por atender al trabajo del curso; esto es, a la resolución de tareas propuestas por el docente. Pareciera que la mayor experiencia en entornos virtuales de este grupo de alumnos favoreció una descentración respecto de la preocupación por el manejo tecnológico del ambiente y una atención más focalizada en las tareas del curso.

No obstante las diferencias mencionadas entre los grupos, es necesario reconocer que las solicitudes de ayuda cuyo contenido está centrado en aspectos relativos al manejo técnico del entorno virtual, se presentan como propias y exclusivas de este tipo de ambientes (Chiecher, 2007).

Cabe destacar, además, que dentro de las demandas planteadas por el Grupo 2 se hallaron solicitudes propias de ese grupo -no planteadas por los estudiantes del Grupo 1- y ligadas al pedido de reprogramación de fechas para las tareas. En estos casos, los alumnos aducían como justificativo de su pedido distintas situaciones personales típicas de un estudiante que decide aprender a distancia (motivos laborales, nacimiento de hijos, problemas de salud, viajes, etc.).

Los pedidos de ayuda presentados en ambos grupos pudieron clasificarse en categorías similares, con excepción de un tipo de solicitud, exclusivo del Grupo 2 
(pedido de reprogramación de tareas). En el Grupo 1, con menor experiencia en el ambiente virtual, se apreció una focalización de la atención -y hasta cierto nivel de ansiedad- respecto del manejo tecnológico del entorno. Avalados por estudios que sostienen que en el ambiente virtual podrían sortearse obstáculos propios de la interacción cara a cara -como por ejemplo, los rasgos de personalidad- (Chiecher, 2007; Kitsantas y Chow, 2007), pensábamos que la inclusión del medio virtual dentro de la propuesta presencial, alentaría la búsqueda de ayuda. Sin embargo, pareciera que la falta de experiencia con la tecnología, la falta de confianza en sí mismos para manejarse con soltura en el ambiente virtual y quizás una escasa autoeficacia percibida en ese medio, incidieron fuertemente sobre el tipo de ayuda que buscaban los estudiantes. Por su parte, el Grupo 2, si bien presentó pedidos de ayuda sobre cuestiones técnicas, en igual medida canalizó demandas relacionadas con las consignas de trabajo; es decir, solicitudes más de tipo instrumental, donde la intención es dominar las tareas y lograr un aprendizaje a partir de su resolución.

Lo que llama poderosamente la atención es que en ninguno de los grupos aparece como frecuente el pedido de ayuda sobre conceptos. Más aún, en ambos fue el tipo de intervención de menor frecuencia. Al respecto, una explicación posible podría estar en que los alumnos del Grupo 1 prefieren quizás consultar sus dudas conceptuales, en caso de tenerlas, en el ámbito presencial o bien en interacción cara a cara con algún compañero. Parece razonable que, de tener posibilidad, se prefiera una explicación ofrecida en el ámbito de la clase, donde uno puede repreguntar y orientar mejor la respuesta del profesor. Respecto del Grupo 2, no contaba con la posibilidad de interacción presencial; sin embargo, las características de estos alumnos -adultos, motivados, autónomos e independientes- pueden contribuir a que persistan por sí mismos en la resolución de las dificultades que se les presentan, sin llegar a hacerlas explícitas en caso de encontrar una solución. Más allá de estas explicaciones posibles, suscribimos al planteo de Arroyo et al. (2004) cuando sostienen que aunque el pedido de ayuda ha sido relacionado positivamente con el aprendizaje, los estudiantes pueden no tener conductas de búsqueda de ayuda óptimas; y algo de esto parece haber sucedido en los dos grupos analizados.

Habría que pensar entonces en estrategias que favorezcan el uso de la búsqueda de ayuda en ambientes virtuales, y muy especialmente, de la formulación de preguntas sobre los conceptos a aprender. Tal vez tareas pensadas con este fin pueden contribuir al logro de este objetivo. Estamos pensando en tareas académicas que más que demandar respuestas, requieran al alumno la formulación de preguntas y su presentación en el foro. 
Para finalizar, coincidimos con Kitsantas y Chow (2007) cuando sostienen que aún no contamos con abundantes estudios sistemáticos que hayan indagado cómo varía la búsqueda de ayuda en ambientes distintos. En efecto, se trata de una importante área de estudio debido al explosivo crecimiento de los cursos virtuales y a los nuevos desafíos que enfrenta la educación para dar instrucción efectiva y soporte a los estudiantes a distancia.

\section{REFERENCIAS BIBLIOGRÁFICAS}

Arroyo, I.; Murray, T.; Woolf, B. P.; Beal, C. (2004). Inferring unobservable learning variables from students' help seeking behavior. [en línea] Disponible en: http://www.springerlink.com/content/ 1tdpxfydwv17nt8x/ [consulta 2009, 2 de Julio]

Blocher, M.; Sujo de Montes, L. (2002). Is online learning for everyone? En: Sposetti, A.; Elstein, S.; Rivero, M. (Comp.) Educacióny nuevastecnologías. Propuestas y experiencias nacionales $e$ internacionales. Argentina: Universidad Nacional de Río Cuarto.

Blocher, M.; Sujo De Montes, L.; Willis, E.; Tucker, G. (2002). Online learning: examining the successful student profile. Journal of Interactive Online Learning. National Centre for Online Learning Research.

Butler, R. (1998). Determinants of helpseeking: relations between perceived reasons for classroom help-avoidance and help-seeking behaviors in an experimental context. Journal of Educational Psychology, vol 90, $\mathrm{n}^{0} 4$, (630-643).

Butler, R.; Neuman, O. (1995). Effects of task and ego achievement goals on helpseeking behaviors and attitudes. Journal of Educational Psychology, vol. 87, $\mathrm{n}^{\circ}$ 2, (261-271).

Chiecher, A. (2007). Aprender en contextos presenciales y virtuales. Motivación, uso de estrategias y percepción del contexto en ambientes presenciales y virtuales. Tesis Doctoral. Doctorado en Psicología. Universidad Nacional de San Luis.

Chiecher, A.; Donolo, D.; Rinaudo, M. C. (2006). Búsqueda de ayuda en ambientes presenciales y virtuales. XIII Jornadas de Investigación. Argentina: Universidad de Buenos Aires.

Chiecher, A.; Donolo, D.; Rinaudo, M. C. (2007). Manejo de recursos en contextos presenciales y virtuales. Un estudio comparativo con dos grupos de estudiantes de posgrado. IV Congreso Nacional y II Internacional de Investigación Educativa. Universidad Nacional de Comahue.

Karabenick, S. (1998). Help seeking as a strategic resource. En: Karabenick, S. Strategic Help Seeking: Implications for Learning and Teaching. Lawrence Erlbaum Associates.

Karabenick, S. (2002). Seeking help in large college classes: a person-centered approach. Contemporany Educational Psychology, vol. 26, $\mathrm{n}^{\mathrm{0}}$ 1, (38-57).

Keefer, J.; Karabenick, S. (1998). Help seeking in the information age. En: Karabenick, S. Strategic Help Seeking: Implications for Learning and Teaching. Lawrence Erlbaum Associates.

Nelson-Le Gall, S. H. (1985). Help seeking behaviours in learning. [en línea] Disponible en: http://eric.ed.gov/ 
ERICDocs/data/ericdocs2sql/content storage $01 / 0000019 \mathrm{~b} / 80 / 2 \mathrm{2f} / 85 / \mathrm{c} 3 . \mathrm{pdf}$ [consulta 2009, 2 de Julio]

Newman, R. (1998). Students Help-seeking during problem solving: influences of personal and contextual achievement goals. Journal of Educational Psychology, vol. 90, $\mathrm{n}^{\circ} 4$, (664-658).

Newman, R.; Schwager, M. (1995). Students Help-seeking during problem solving: effects of grade, goal and prior achievement. American Educatinal Research Journal, vol. 32, $\mathrm{n}^{\mathrm{O}}$ 2, (352376).

Pintrich, P.; Smith, D.; García, T.; Mckeachie, W. (1991). A Manual for the Use of the Motivated Strategies for Learning Questionnaire (MSLQ). National Center for Research to Improve Postsecondary Teaching and Learning. University of Michigan.

Puustinen, M. (1998). Help-seeking behavior in a problem-solving situation: developmentofself-regulation.European Journal of Psychology of Education, vol. XIII, $\mathrm{n}^{0}$ 2, (271-282).

Rinaudo, M. C.; Donolo, D.; Chiecher, A. (1999). Los procesos de solicitar, dar y recibir ayuda pedagógica en el ámbito de las clases universitarias. Cronía, Revista de Investigación de la Facultad de Ciencias Humanas, año 3 , vol. $3, \mathrm{n}^{\mathrm{o}}$ $1,(60-70)$.
Ross, J.; Cousins, J. (1995). Giving and receiving explanations in cooperative learning groups. The Alberta Journal of Education Research, vol. XLI, n ${ }^{0} 1,\left(103^{-}\right.$ 121).

Ryan, A.; Pintrich, P.; Midgley, C. (2001). Avoiding seeking help in the classroom: who and why? Educational Psychology Review, vol. 13, $\mathrm{n}^{0}$ 2, (93-114).

Suárez, J. M.; Anaya, D. (2004). Educación a distancia y presencial: diferencias en los componentes cognitivo y motivacional de estudiantes universitarios. RIED, vol. $7,1 / 2,(65-75)$.

Taplin, M.; Yum, J.; Jegede, O.; Fan, R.; Chan, M. (2001). Help seeking strategies used by high-achieving and low achieving distance education students. Journal of Distance Education, vol. 16.

Tucker, G.; Blocher, J. (2002). Collaborative learning and the online learner: Do those who choose online delivery want collaborative learning? Proceedings of the $13^{\text {th }}$ International Conference of the Society for Information Technology and Teacher Education. Nashville, Tennessee.

Van Der Meij, H. (1990). An experimental study of the when and what of children's questions. Tijdschrift voor Onderwijsresearch, $\mathrm{n}^{0} 3,(162-171)$. 


\section{PERFIL ACADÉMICO DE LOS AUTORES}

Analía Chiecher. Doctora en Psicología por la Universidad Nacional de San Luis; Magíster en Educación y Universidad y Licenciada en Psicopedagogía por la Universidad Nacional de Río Cuarto. Investigadora asistente de CONICET. Autora de artículos y libros sobre aprendizaje en ambientes presenciales y virtuales.

E-mail achiecher@hum.unrc.edu.ar

Danilo Donolo. Doctor en Ciencias de la Educación y Profesor Titular en asignaturas del Dpto. de Ciencias de la Educación, Universidad Nacional de Río Cuarto. Investigador independiente de CONICET. Autor de libros y artículos sobre educación. Director de Proyectos de Investigación aprobados por CONICET y ANPCYT.

E-maildonolo@hum.unrc.edu.ar

María Cristina Rinaudo. Doctora en Ciencias de la Educación y Profesora Titular en asignaturas del Dpto. de Ciencias de la Educación, Universidad Nacional de Río Cuarto. Directora de Proyectos de Investigación aprobados por CONICET y ANPCYT.

E-mail crinaudo@hum.unrc.edu.ar

DIRECCION DE LOS AUTORES

Juan B. Justo 925,

Río Cuarto, Córdoba,

Argentina. CP 5800

Fecha de recepción del artículo: 06/10/08

Fecha de aceptación del artículo: 03/12/08 